\title{
Tracing the Quenching History of Cluster Galaxies in the EAGLE Simulation
}

\author{
Diego Pallero $^{1 \star}$, Facundo A. Gómez ${ }^{1,2}$, Nelson D. Padilla ${ }^{3,4}$, S. Torres-Flores ${ }^{1}$, \\ R. Demarco ${ }^{5}$, P. Cerulo 5 and D. Olave-Rojas ${ }^{6}$ \\ ${ }^{1}$ Departamento de Física y Astronomía, Universidad de La Serena, Av. Juan Cisternas 1200 Norte, La Serena, Chile \\ ${ }^{2}$ Instituto de Investigación Multidisciplinar en Ciencia y Tecnología, Universidad de La Serena, Raúl Bitrán 1305, La Serena, Chile \\ ${ }^{3}$ Instituto de Astrofísica, Pontificia Universidad Católica de Chile, Santiago, Chile \\ ${ }^{4}$ Centro de Astro-Ingeniería, Pontificia Universidad Católica de Chile, Santiago, Chile \\ ${ }^{5}$ Departamento de Astronomía, Facultad de Ciencias Físicas y Matemáticas, Universidad de Concepción, Concepción, Chile \\ ${ }^{6}$ Departamento de Física, Facultad de Ciencias, Universidad del Bío Bío, Collao 1202, Concepción, Chile
}

Accepted XXX. Received YYY; in original form ZZZ

\begin{abstract}
We use the EAGLE hydrodynamical simulation to trace the quenching history of galaxies in its 10 most massive clusters. We use two criteria to identify moments when galaxies suffer significant changes in their star formation activity: $i$ ) the instantaneous star formation rate (SFR) strongest drop, $\Gamma_{\mathrm{SFR}}^{\mathrm{SD}}$, and ii) a "quenching" criterion based on a minimum threshold for the specific SFR of $\lesssim 10^{-11} \mathrm{yr}^{-1}$. We find that a large fraction of galaxies $(\gtrsim 60 \%)$ suffer their $\Gamma_{\mathrm{SFR}}^{\mathrm{SD}}$ outside the cluster's $\mathrm{R}_{200}$. This "pre-processed" population is dominated by galaxies that are either low mass and centrals or inhabit low mass hosts $\left(10^{10.5} \mathrm{M}_{\odot} \lesssim \mathrm{M}_{\text {host }} \lesssim 10^{11.0} \mathrm{M}_{\odot}\right)$. The host mass distribution is bimodal, and galaxies that suffered their $\Gamma_{\mathrm{SFR}}^{\mathrm{SD}}$ in massive hosts $\left(10^{13.5} \mathrm{M}_{\odot} \lesssim \mathrm{M}_{\text {host }} \lesssim 10^{14.0} \mathrm{M}_{\odot}\right)$ are mainly processed within the clusters. Pre-processing mainly limits the total stellar mass with which galaxies arrive in the clusters. Regarding quenching, galaxies preferentially reach this state in high-mass halos $\left(10^{13.5} \mathrm{M}_{\odot} \lesssim \mathrm{M}_{\text {host }} \lesssim 10^{14.5} \mathrm{M}_{\odot}\right)$. The small fraction of galaxies that reach the cluster already quenched has also been preprocessed, linking both criteria as different stages in the quenching process of those galaxies. For the $z=0$ satellite populations, we find a sharp rise in the fraction of quenched satellites at the time of first infall, highlighting the role played by the dense cluster environment. Interestingly, the fraction of pre-quenched galaxies rises with final cluster mass. This is a direct consequence of the hierarchical cosmological model used in these simulations.
\end{abstract}

Key words: galaxies:clusters: general - galaxies: evolution - galaxies: formation galaxies: star formation - galaxies: haloes

\section{INTRODUCTION}

Since the first half of the twentieth century, it has been known that colors reflect the predominant stellar populations in galaxies and that they are related to their morphology (Morgan \& Mayall 1957). The colour-morphology (Roberts \& Haynes 1994) and color-magnitude relations (Chester \& Roberts 1964; Faber 1973) are now widely used to study the properties of galaxies. As a result, rather than selecting objects according to their early- or late-type mor-

^ E-mail:dpallero@dfuls.cl phology, galaxies can be separated between red and blue, which naturally relates with their star formation and metalenrichment history. Studies in the local universe show that, in general, galaxies present a strong bimodal color distribution (Strateva et al. 2001; Baldry et al. 2006; Cassata et al. 2008), regardless of the environment in which they reside (Hogg et al. 2004; Baldry et al. 2006). Reproducing this bimodality, and understanding the role played by the environment, has become an important goal for galaxy-evolution theories (Trayford et al. 2015; Nelson et al. 2018).

One of the first indications that the environment plays a fundamental role in driving the evolution of galaxies was 
the morphology-density relation (Dressler 1980, 1984). Observational studies have shown that in high density environments there is a greater fraction of galaxies with early-type morphology than in low density environments, and that the fraction of early-type galaxies in clusters rises toward the cluster's center (Brough et al. 2017; Cava et al. 2017).

In addition, several studies during the last decades have shown that dense environments can also affect the star formation history of galaxies (Gunn \& Gott 1972; Dressler 1980; Moore et al. 1996; Poggianti et al. 2001; Boselli et al. 2005). Naturally, the cores of galaxy clusters are an ideal laboratory to study how the environment affects the evolution of galaxies in dense regions and at different redshifts (Cayatte et al. 1990; Smail et al. 1997; Bravo-Alfaro et al. 2000; Boselli et al. 2005). Evidence of global transformations for galaxies over time is given by the increasing fraction of spiral in clusters up to $\mathrm{z} \sim 0.5$ (Dressler et al. 1997; Fasano et al. 2000; Desai et al. 2007), and thanks to the fact that high-z clusters are observed to contain more star-forming galaxies compared to present-day (Butcher \& Oemler 1984; Poggianti et al. 2006).

It is also well known that there are differences between the properties of galaxies located in the inner and outer regions of galaxy clusters. Some authors (Kodama et al. 2001; Treu et al. 2003), suggest that this is the result of a variety of mechanisms that act at different distances from the cluster center, driving galaxy evolution with different timescales. Moreover, it has been observed (e.g. Dressler et al. 2013; Hou et al. 2014; Bianconi et al. 2018) that in the outskirts of clusters infalling galaxy partly distribute in the form of groups.

Theoretical works (e.g. McGee et al. 2009; De Lucia et al. 2012) suggest that $\sim 25-40 \%$ of galaxies belonging to a massive cluster $\left(\mathrm{M}_{\text {halo }} \sim 10^{14.5}-10^{15.0}\left[\mathrm{M}_{\odot}\right]\right)$ at $\mathrm{z}=0$ have been accreted in such groups. For the aforementioned reasons, the study of galaxy properties in cluster outskirts, where these systems are still assembling, has attracted the interest of several astronomers (e.g. Just et al. 2010; Cybulski et al. 2014; Jaffé et al. 2016). In particular, some authors (e.g. Hou et al. 2014; Haines et al. 2015; Bianconi et al. 2018) have studied the variation of the fraction of quiescent galaxies with the projected distance from the centers of clusters, finding that out to $3 R_{200}$ clusters are richer in passive galaxies than the field. These results can only be explained if star formation was quenched in galaxies prior to their accretion on to clusters, when they were still members of in-falling groups (pre-processing Zabludoff \& Mulchaey 1998; Fujita 2004). The fact that galaxies are "preprocessed" in groups before their accretion on to clusters shows that groups of galaxies constitute an important piece in the physics of galaxy formation and evolution, because the processes that take place within them may significantly alter star formation and change the structural and chemical properties of galaxies. Groups provide, then, further laboratories to study the environmental drivers of galaxy evolution (e.g. Dressler et al. 2013; Bianconi et al. 2018; Olave-Rojas et al. 2018).

At $\mathrm{z} \sim 0$, the specific star-formation rate of galaxies in dense environments is significantly smaller than in lower density regions (Hashimoto et al. 1998; Lewis et al. 2002; Kauffmann et al. 2004; Gray et al. 2004; Balogh et al. 2007). Additionally, higher fractions of quiescent or passive galax- ies are found in dense regions (Poggianti et al. 1999; Baldry et al. 2006; van den Bosch et al. 2008; Gavazzi et al. 2010; Haines et al. 2013). These studies also provide evidence that the star-forming activity and galaxy morphology can be correlated with $\mathrm{z} \sim 0$ galactic stellar mass. Less massive galaxies also are more susceptible to environmental effects, indicating that the quenching of star formation can be accelerated in dense environments(De Lucia et al. 2012; Muzzin et al. 2012; Jaffé et al. 2016).

In spite of the observed relation between environment and cessation of star formation activity, i.e. "environmental quenching", it is important to take into account internal process that can drive galaxy quenching. This process, known as "mass quenching" or "internal quenching", can arise as a result of, e.g. internal gas consumption, supernova and AGN feedback, star formation feedback or halo gas heating (see e.g. Peng et al. 2010, Efstathiou 2000, Croton et al. 2006, Dekel \& Birnboim 2008, Cantalupo 2010). The dominance of one way over the other is where the dichotomy of "nature versus nurture" was born, and has been one of the main subjects of study for extragalactic astronomy in the last years.

According to Oesch et al. (2016), quenching may start shortly after the first appearance of the galaxies, at roughly $\mathrm{z} \sim 11$, but the environment does not play an important role until $\mathrm{z} \sim 1.6$, with the environmental quenching efficiency rising by a factor of $\sim 3.5$ between $\mathrm{z} \sim 1.6$ and $\mathrm{z}$ $\sim 0.9$. The quenching efficiency is defined as the ratio between passive galaxies in clusters and passive galaxies in the field, quantifying the number of galaxies that would be starforming if they were in the field (Peng et al. 2010, 2012; Nantais et al. 2016). Nevertheless, a study of the sSFR and the fraction of star-forming galaxies in clusters at $\mathrm{z} \sim 1$ from the GCLASS survey (Muzzin et al. 2012) shows that mass quenching dominates over environmental quenching, at least at this redshift. Balogh et al. (2016), using another cluster sample from GCLASS, found that the mechanisms driving the quenching at $\mathrm{z}>1$ may be different from those at $\mathrm{z} \sim 0$. On the one hand, at high redshift, the cessation of star formation is mainly driven by a combination of gas consumption (due to an enhancement of star formation) and gas outflows as a result of supernovae and AGN feedback. On the other hand, at low redshift, dynamical mass removal mechanisms (due to environment) may be the main driver for the quenching of galaxies in clusters.

A detailed description of the main mechanisms that lead to environmental quenching is provided by Boselli \& Gavazzi (2006) and Jaffé et al. (2016). They separate these mechanisms in three broad categories:

- Gravitational interactions between galaxies: Mergers can change drastically the star formation history of galaxies, as well as their morphology and kinematics. This phenomenon is usually observed in low-density environment such as groups of galaxies. In high-density environments, galaxies can experience harassment from other cluster members, through fast and aggressive encounters (Toomre \& Toomre 1972; Barnes \& Hernquist 1996; Walker et al. 1996; Moore et al. 1999);

- Interactions between galaxies and the intra-cluster medium: Ram-pressure from the intracluster medium can strip the gas of the galaxies and remove their interstellar medium (Gunn \& Gott 1972; Abadi et al. 1999; Quilis et al. 

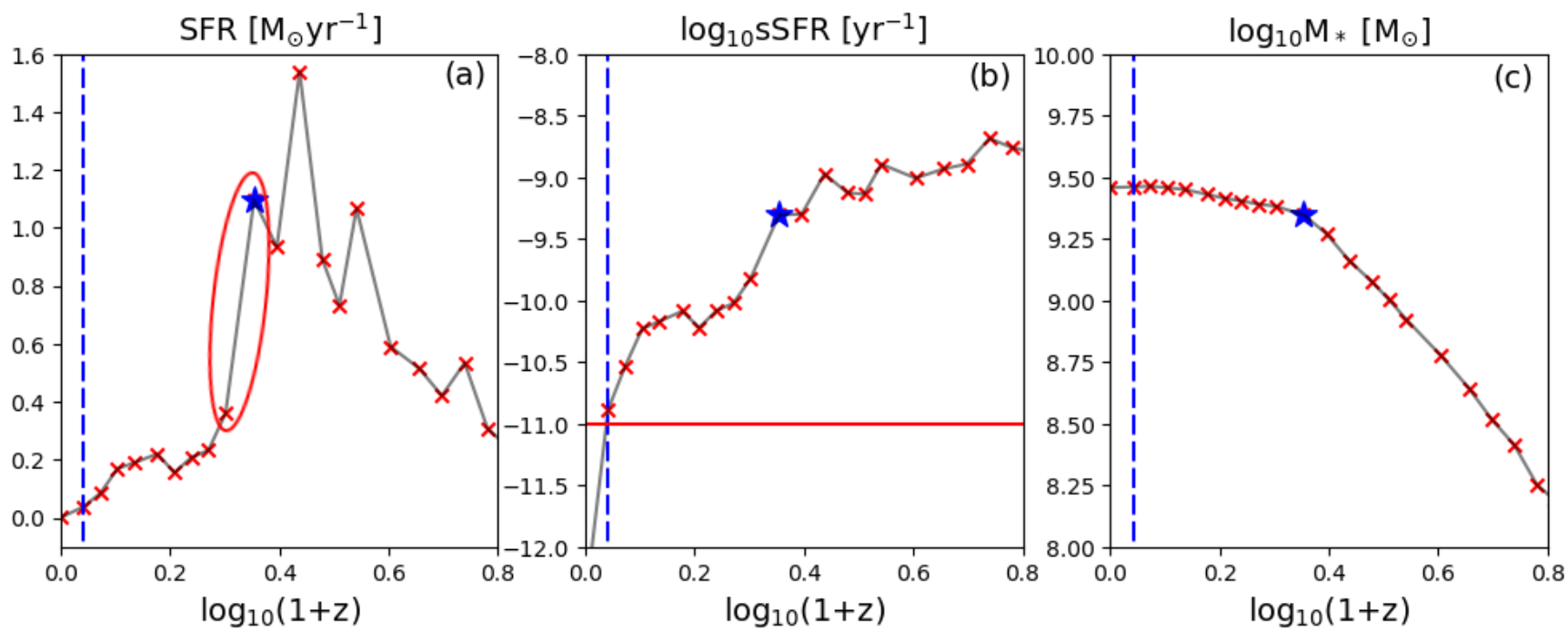

Figure 1. Examples of the selection criteria used in this work to determine whether a galaxy was processed or quenched, for a random galaxy in our sample. Panel (a) shows the star formation rate against redshift. The red ellipse highlights the strongest drop in the star formation activity, and the blue dashed line corresponds to the time of the first infall into the final cluster's $R_{200}$. Panel (b) shows the specific star formation rate of the galaxy against redshift. The red solid line shows the critical star formation rate imposed by our selection criterion to define quenched galaxies. The blue dashed line corresponds to the first infall into $\mathrm{R}_{200}$. Panel (c) shows the growth of the galaxy stellar mass through cosmic time. The blue star indicates the moment when the processing started. We can see that the growth of the stellar mass is suppressed after the strongest drop, and that the specific star formation rate decreases abruptly after passing $R_{200}$.

2000; Vollmer et al. 2001; Jaffé et al. 2015; Benítez-Llambay et al. 2013);

- Gravitational interactions between clusters and galaxies: The tremendous gravitational potential of the cluster can perturb some observable properties of the members, inducing gas inflows, forming bars, compressing the gas or concentrating the star formation (Miller 1986; Byrd \& Valtonen 1990; Boselli \& Gavazzi 2006).

It is precisely because of this complex nature of the environmental quenching that it is difficult to separate the aforementioned processes. It is expected that, at least, some of these processes act simultaneously and that they are effective in different overlapping regions of the cluster. Some studies show that the effectiveness of these processes is linked to the galaxy's cluster-centric distance (Moran et al. 2007).

A good approach to study the mechanisms that impact galaxy evolution is through cosmological models (Fujita 2004; Wetzel et al. 2013; Vijayaraghavan \& Ricker 2013; Schaye et al. 2015; Nelson et al. 2018). Several works have used simulations to understand the properties of galaxies in different environments, and how their evolutionary history results in changes of their properties such as colors, stellar mass and star formation rate (Trayford et al. 2015, 2016; Katsianis et al. 2017; Tescari et al. 2018; Nelson et al. 2018). Hydrodynamical simulations can be used to define and test different criteria that can be used to understand the processes that drive galaxies to be quenched. Simulations also allow us to follow the evolution of galaxies in different environments and the evolution of their properties from $\mathrm{z} \sim$ 20 to $\mathrm{z}=0$. Since clusters at $\mathrm{z}>1.5$ are difficult to detect, due to the fact that they are still in an assembling process, simulations are a helpful tool to study the role that the environment plays at such high redshift (see e.g. Overzier 2016).

In this paper, we use the public database from the stateof-the-art EAGLE hydrodynamic simulations (Schaye et al. 2015; Crain et al. 2015; McAlpine et al. 2016) to trace the evolution history of the satellite galaxies that belong to the ten most massive clusters at $\mathrm{z} \sim 0$. We aim at identifying the environment in which galaxies preferentially cease their star formation and signatures that could be used to determine the main physical mechanism leading to the cessation of star formation of cluster satellite galaxies. We compare the results obtained from two different criteria to identify when star formation in galaxies significantly drops. The hydrodynamic simulations of the EAGLE project are perfectly suited for this study since they provide the possibility to study the evolution of galaxies and their properties.

This paper is organized as follows: in Section 2 we describe the EAGLE simulation, its main characteristics and the main potentialities that it provides for this study. In Section 3 we define the two criteria used in this work to locate those moments when galaxies suffer an important variation in their star formation; in Section 4 we describe the results obtained using our two approaches, putting special interest on the environment where these events take place. Finally, in Section 5 we summarize our main conclusions and compare our results with both observational and theoretical works. A brief discussion of some future projects are presented in this section as well. 


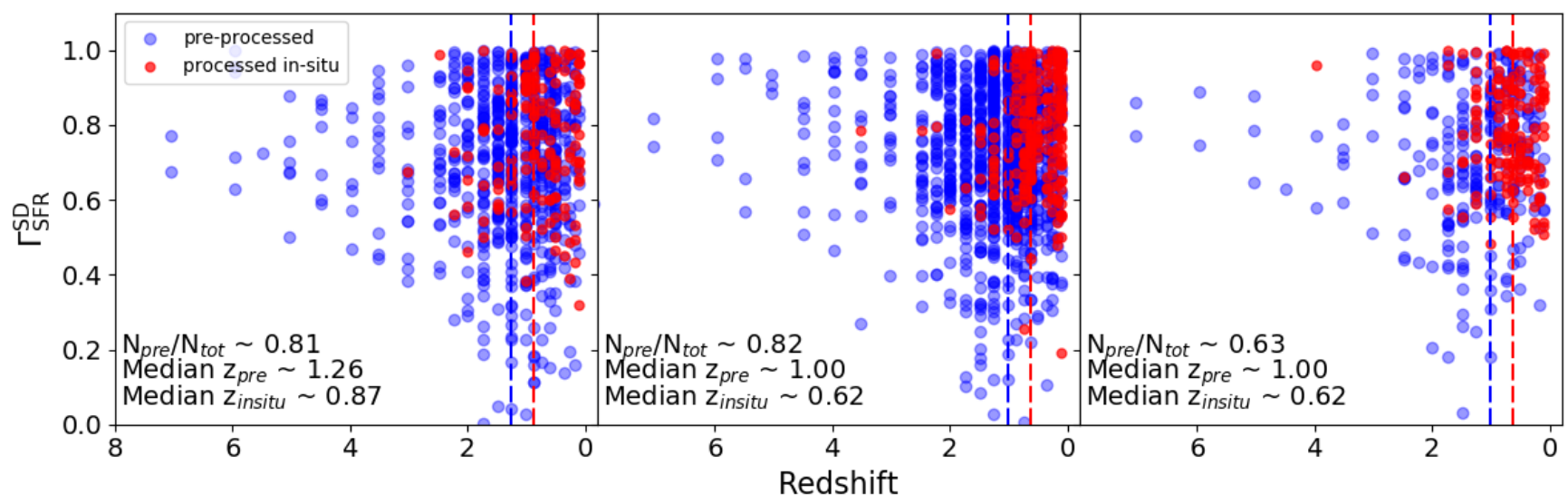

Figure 2. Distribution of $\Gamma_{\mathrm{SFR}}^{\mathrm{SD}}$ (normalized instantaneous strongest drop of the SF activity), as a function of the redshift at which it takes place. Blue dots correspond to galaxies that suffer their $\Gamma_{\mathrm{SFR}}^{\mathrm{SD}}$ outside the cluster's $\mathrm{R}_{200}$ (pre-processed), while red dots to those that suffer their $\Gamma_{\mathrm{SFR}}^{\mathrm{SD}}$ inside $\mathrm{R}_{200}$ (processed in-situ). The medians for both samples are indicated by the dashed lines. The panels are organized from left to right as high-mass clusters $\left(14.6<\log _{10} \mathbf{M}_{\text {host }}\left[\mathrm{M}_{\odot}\right]<14.8\right)$, intermediate-mass clusters $\left(14.3<\log _{10} \mathrm{M}_{\text {host }}\left[\mathrm{M}_{\odot}\right]<14.6\right)$ and low-mass clusters $\left(14.0<\log _{10} \mathrm{M}_{\text {host }}\left[\mathrm{M}_{\odot}\right]<14.3\right)$, respectively.

\section{THE EAGLE SIMULATION}

The EAGLE project, is a suite of cosmological hydrodynamical N-body simulations. These simulations were run with a modified version of the GADGET-3 code, wich is an improved version of GADGET-2 (Springel 2005). All the simulations adopt a flat $\Lambda \mathrm{CDM}$ cosmology whose parameters were calibrated with the data obtained by the Planck mission (Planck Collaboration et al. 2014); $\Omega_{\Lambda}=0.693, \Omega_{m}=$ $0.307, \Omega_{b}=0.04825, \sigma_{8}=0.8288, \mathrm{Y}=0.248$ and $\mathrm{H}_{0}=67.77$ $\mathrm{km} \mathrm{s}^{-1}$.

In particular, for this work we select our sample of galaxies from the main simulation, referred to as L100N1504, which consists of a periodic box with a volume of $(100 \mathrm{cMpc})^{3}$, initially containing $1,504^{3}$ gas particles with an initial mass of $1.81 \times 10^{6} \mathrm{M}_{\odot}$, and the same amount of dark matter particles with a mass of $9.70 \times 10^{6} \mathrm{M}_{\odot}$.

Each simulation counts with 29 discrete snapshots from redshift 20 to 0 , with a time span between consecutive snapshots ranging from 0.3 to $1 \mathrm{Gyr}$. Radiative cooling and photoheating are implemented following Wiersma et al. (2009a), assuming an optically thin X-Ray/UV background (Haardt \& Madau 2001). Star formation is implemented stochastically following Schaye \& Dalla Vecchia (2008), and using the metallicity-dependent density threshold shown in Schaye (2004). This reproduces the observed Kennicutt-Schmith law (Kennicutt 1998). Each particle is assumed to be a single-age stellar population, with a Chabrier initial mass function in the range $0.1 \mathrm{M}_{\odot}-100 \mathrm{M}_{\odot}$ (Chabrier 2003).

Stellar evolution is modelled as shown in Wiersma et al. (2009b), and chemical enrichment is followed for the 11 elements that most contribute to radiative cooling from massive stars (Type II supernovae and stellar winds) and intermediate-mass stars (Type Ia supernovae and AGB stars). Following Dalla Vecchia \& Schaye (2012), the thermal-energy product of stellar feedback is stochastically distributed among the gas particles surrounding the event without a preferential direction.

The EAGLE project calibrated the free parameters as- sociated with stellar feedback to match the observations for the stellar mass function in a range of $10^{8} \mathrm{M}_{\odot}-10^{11} \mathrm{M}_{\odot}$ and the size-mass relation for galaxies in a range of $10^{9} \mathrm{M}_{\odot}$ $10^{11} \mathrm{M}_{\odot}$ (Schaye et al. 2015; Furlong et al. 2015, 2017). The appropriate calibration of the subgrid physics and the good agreement with the observational data make these simulations our best tool to study the evolution in the star formation of galaxies in these mass ranges for different environments.

The halo catalogues provided in the public database (used in this work) were built using a friend-of-friends (FoF) algorithm which identifies dark matter overdensities following Davis et al. (1985), considering a linking length of 0.2 times the average inter-particle spacing. Baryonic particles are assigned to the FoF halo of their closest dark matter particle. Subhalo catalogues were built using the SUBFIND algorithm (Springel et al. 2001; Dolag et al. 2009), which identifies local overdensities using a binding energy criterion for particles within a FoF halo. We will define as galaxies those structures recognized by the SUBFIND algorithm (Springel et al. 2001; Dolag et al. 2009) which posses a total stellar mass greater than $10^{8} \mathrm{M}_{\odot}$ and a total mass greater than $10^{9}$ $\mathrm{M}_{\odot}$. These masses are obtained by direct summation of the corresponding particles; i.e., particles bound to the subhalo according to the SUBFIND. Since the simulations considered in this work have a baryonic mass resolution of $1.6 \times 10^{6}$ $\mathrm{M}_{\odot}$ and dark matter mass resolution of $9.1 \times 10^{6} \mathrm{M}_{\odot}$, we ensure that we have at least 100 baryonic and dark matter particles in each galaxy, thus avoiding spurious results and non-physical detections. The analyzed clusters correspond to the 10 most massive in the simulation at $z=0$. They all posses $\mathrm{M}_{200}>10^{14} \mathrm{M}_{\odot}$. A galaxy is defined as satellite if it can be found inside the host $R_{200}$ at $z=0$. 


\section{THE END OF THE STAR FORMING PHASE: DEFINITIONS}

According to Peng et al. (2010), the quenching of a galaxy is the result of a process with two different components. A continuous component associated with internal galactic processes such as star formation and AGN feedback, and a "once-only" component due to environmental processes. Note, however, that other mechanisms like mergers may also have an important effect on the star formation activity.

To determine the moment when the star formation activity in a galaxy drops in a significant way, two different criteria are introduced: one based on the maximum drop of the SFR between two consecutive snapshots of the simulation, and the other based on a minimum threshold for specific star formation rate (sSFR). The first criterion seeks to identify those mechanisms that abruptly reduce star formation in galaxies, while the second one is meant to define when a galaxy is actually quenched, that is, it is no longer forming stars (e.g. Weinmann et al. 2010; De Lucia et al. 2012; Wetzel et al. 2012). The aim of using these two criteria is to determine and understand the different stages of quenching and how they are affected by the environment. From now on, we will refer to a galaxy as "processed" when it suffers its strongest drop, whereas we will refer to a galaxy as "quenched" when it reaches the imposed threshold in sSFR.

\subsection{SFR Strongest Drop}

One of our goals is to identify the mechanisms that can abruptly reduce the star formation in galaxies. For this purpose, we first calculate for each galaxy the variation of the star formation rate between two consecutive snapshots in the simulation, normalized by the star formation in the earliest snapshot. This is

$\Gamma_{\mathrm{SFR}}=\frac{\mathrm{SFR}_{i+1}-\mathrm{SFR}_{i}}{\mathrm{SFR}_{i}}$,

where the subscript $i$ indicates the simulation snapshot, and $i+1$ is at a lower redshift than $i$. $\Gamma_{\mathrm{SFR}}$ is computed only if the difference between the SFR value in the two snapshots is larger than $1 \times 10^{-3} \mathrm{M}_{\odot} \mathrm{yr}^{-1}$. This constraint was imposed to avoid measures of $\Gamma_{\mathrm{SFR}}$ for galaxies already quenched. fWe then define $\Gamma_{\mathrm{SFR}}^{\mathrm{SD}}$ as the fraction of star formation lost at the moment when the strongest drop occurs, i.e.

$\Gamma_{\mathrm{SFR}}^{\mathrm{SD}}=\left|\min \Gamma_{\mathrm{SFR}}\right|$

We refer to this method as the "Strongest drop selection criterion". $\Gamma_{\mathrm{SFR}}^{\mathrm{SD}}$ takes into account those episodes when a "once-only" event affects the star formation activity of the galaxies but does not take into account any rejuvenation scenario that could take place afterwards. For this reason, it is not a good tracer of definitive quenching. However, the information gathered by this criterion allows us to find the epochs at which the galaxy suffers a "processing" event, in particular the most significant one. An example of this selection criterion is shown in Figure 1, panel (a), where we plot, as a function of time, the star formation rate of a random galaxy in our sample. The red ellipse highlights the moment when $\Gamma_{\mathrm{SFR}}^{\mathrm{SD}}$ takes place. In particular for this galaxy, the $\Gamma_{\mathrm{SFR}}^{\mathrm{SD}}$ is the result of several processes that heat and remove its cold gas content, producing a stagnation in the evolution of the stellar mass and a small decrease in the total gas mass of the galaxy. Unfortunately, we cannot isolate the different mechanisms that produce this processing event due to the lack of temporal resolution. We will further explore this in a future work using a better suited simulation.

\subsection{Critical sSFR criterion}

We further wish to define a criterion that aims at identifying the moment when the galaxies reach a definitive state of "quenching". Several different definitions of "quenched galaxy" have been proposed in the literature. Here we used the criterion used in Wetzel et al. (2013). According to this criterion, a galaxy can be considered effectively quenched once it reaches a $\mathrm{sSFR}^{\mathrm{Q}}=10^{-11} \mathrm{yr}^{-1}$. At this point the galaxy is considered to be passive. From now on we will refer to those galaxies with a sSFR lower than $\mathrm{sSFR}^{\mathrm{Q}}$ as "quenched galaxies", and we will call this selection criterion the "Critical sSFR Selection Criterion". When using this semi-observational definition, we will only focus on galaxies that are quenched at redshift $z=0$. This is to ensure that the selected galaxies will not suffer a rejuvenation process during their evolution. From each of our quenched galaxies, we will extract information about the environment and the time when the quenching state is reached.

An example of this selection criterion is shown in Figure 1, panel (b), where the sSFR is shown for the same galaxy from the previous example as a function of time. The red line indicates the sSFR threshold established in previous works (Weinmann et al. 2010; De Lucia et al. 2012; Wetzel et al. 2012, 2013) for passive galaxies. In particular for the galaxy shown in the example, the critical star formation is reached once it crosses the $R_{200}$ of the cluster for the first time, showing the importance of dense environments in the quenching of star formation.

\section{RESULTS}

We wish to study the dependencies of star formation quenching on environmental and internal processes focusing on dense environments such as those that can be found in galaxy clusters. For this it is necessary to characterize the properties of individual galaxies such as stellar mass, sSFR and total mass, as well as the overall properties of the host cluster such as total mass and virial radius. We will study how these properties evolve as a function of time, and focus on those moments where individual galaxies experience sharp falls in their star formation rates.

As previously discussed in Section 1, in this work we focus on the population of galaxies associated with the 10 most massive clusters of the EAGLE simulations. In order to study properties of these galaxies as a function of clusters mass with better statistics, the clusters were stacked in three different bins of $z=0$ total mass:

- high mass: $14.6<\log _{10} \mathrm{M}_{\text {host }}\left[\mathrm{M}_{\odot}\right]<14.8$,

- intermediate mass: $14.3<\log _{10} \mathrm{M}_{\text {host }}\left[\mathrm{M}_{\odot}\right]<14.6$,

- low mass: $14.0<\log _{10} \mathrm{M}_{\text {host }}\left[\mathrm{M}_{\odot}\right]<14.3$.

We will refer to these three categories as HMC, IMC and LMC, respectively. The numbers of cluster that fall in each 
(a)

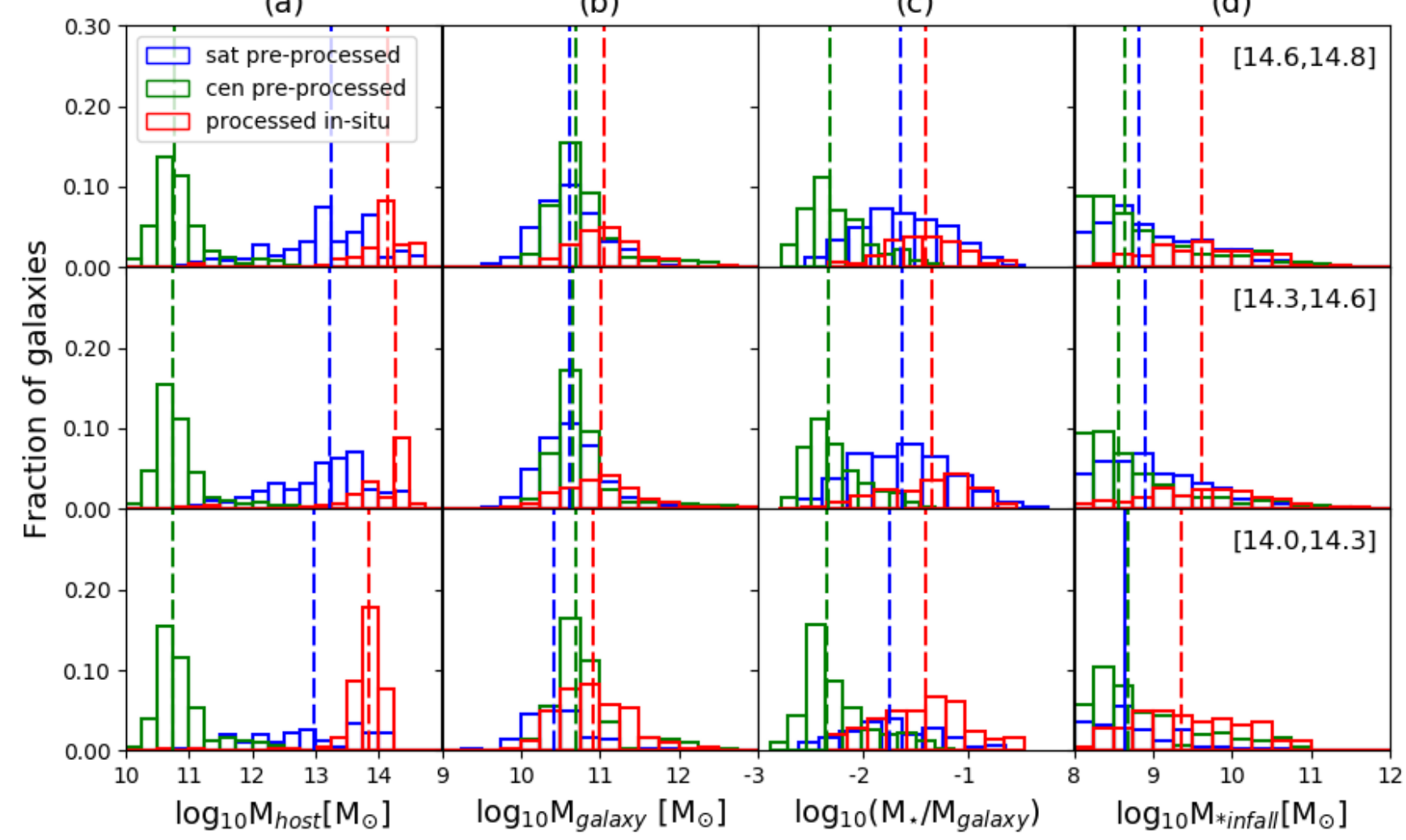

Figure 3. Mass distribution of galaxies and their hosts at key moments related to the $\Gamma_{\mathrm{SFR}}^{\mathrm{SD}}$. Each row shows the results obtained after stacking the distribution of galaxies associated with clusters within different mass ranges. Column (a) shows the mass distribution of the host of each galaxy at the moment of their $\Gamma_{\mathrm{SFR}}^{\mathrm{SD}}$. Column (b) shows the total mass distribution of the galaxies at their $\Gamma_{\mathrm{S} F \mathrm{~S}}^{\mathrm{S}}$. Column (c) shows the stellar mass fraction distributions at $\Gamma_{\mathrm{SFR}}^{\mathrm{SD}}$. Column (d) shows the stellar mass distribution of galaxies at the time of their first infall into the cluster they belong to at $z=0$. Blue, red and greed bars correspond to galaxies pre-processed, in-situ processed, and processed as centrals, respectively. The dashed lines indicate the median of each distribution.

bin are 2 for the HMC, 5 for the IMC and 3 for the LMC. In this section we present our results based on the two previously defined criteria to identify the time at which the star formation activity of a galaxy is significantly altered. We will use the terms pre and in-situ for galaxies that suffer the previously described processes inside or outside the cluster $R_{200}$, respectively.

\subsection{Strongest Drop Selection Criterion}

We first focus on abrupt changes in the SF activity. We start by computing $\Gamma_{\text {SFR }}^{\mathrm{SD}}$ for all galaxies that belong to the 10 most massive clusters at $z=0$. Our goal is to assess where and when they suffer their most significant processing event. The total number of galaxies in the HMC, IMC and LMC bins are $\mathrm{N}_{\text {gal }}=846, \mathrm{~N}_{\text {gal }}=1430$ and $\mathrm{N}_{\text {gal }}=421$, respectively. Note that the differences in the number of galaxies is mainly due to the number of clusters that fall in each mass bin.

In Figure 2 we show $\Gamma_{\text {SFR }}^{\text {SD }}$ for all galaxies as a function of the redshift at which this event takes place. The blue and red dots correspond to pre- and in-situ processed galaxies, respectively. The different panels show the results for the different mass bins. We can clearly see that, for the pre-processed population, there is no preferential redshift for $\Gamma_{\mathrm{SFR}}^{\mathrm{SD}}$ to take place. Note as well that there is no clear correlation between redshift and the typical value of $\Gamma_{\mathrm{SFR}}^{\mathrm{SD}}$ for both populations. This indicates that these "once-only" events that significantly affect star formation activity are not associated with any preferential epoch.

In all mass bins, the majority of the galaxies have been pre-processed. Interestingly, for in-situ processed galaxies, $\Gamma_{\text {SFR }}^{\text {SD }}$ typically occurs at lower values of redshift than for preprocessed galaxies. This can be seen from the dashed vertical lines, which indicate the median redshift for each population. Note as well that the pre-processed fraction grows with clusters mass, but the median in redshift for pre-processing remains the same regardless of the mass bin. This shows that, although more massive clusters accrete a greater number of pre-processed galaxies, the redshift at which $\Gamma_{\text {SFR }}^{\text {SD }}$ typically takes place is independent of the $z=0$ mass of the clusters in which galaxies reside.

To understand how the processing affects the evolution of galaxies and which is the role played by the environment, we characterize the mass distribution of the hosts in which these galaxies resided when they suffered their $\Gamma_{\mathrm{SFR}}^{\mathrm{SD}}$. In Figure 3, panels a), we show the fraction of galaxies per bin of host halo mass, $\mathrm{M}_{\text {host }}$, at the time of $\Gamma_{\mathrm{SFR}}^{\mathrm{SD}}$. Fractions are expressed with respect to the total galaxy sample. We split our 
(a)

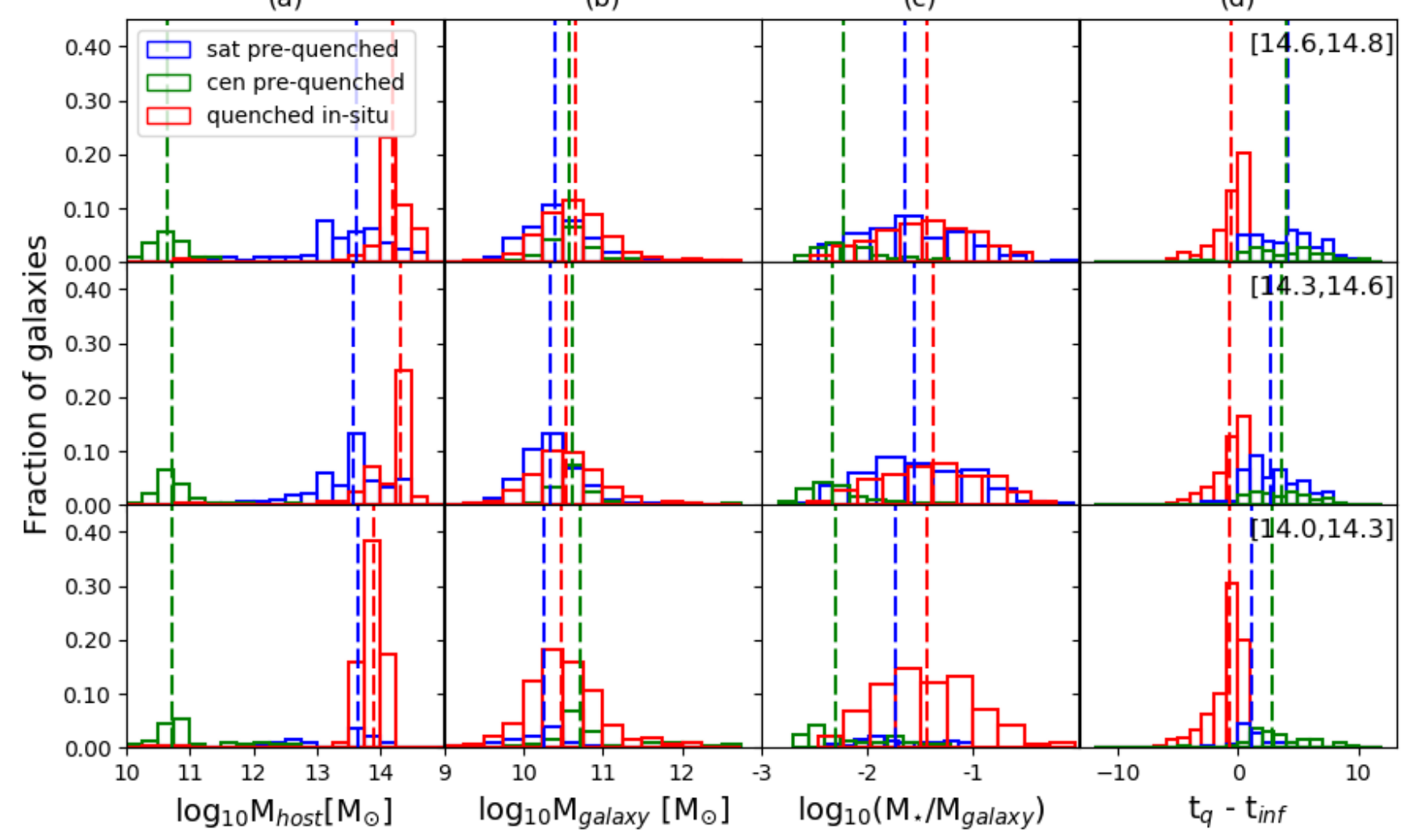

Figure 4. Mass distribution of galaxies and their hosts at the moment when they reach their quenching state. Each row shows the results obtained after stacking the distribution of galaxies associated with clusters within different mass ranges. Column (a) shows the mass distribution of the host of each galaxy. Column (b) shows distribution of galaxies' total mass. Column (c) shows the stellar mass fraction distributions. Column (d) shows the distribution of times, in lookbacktime, at which galaxies become quenched. Blue, red and greed bars correspond to galaxies pre-quenched, in-situ quenched, and quenched as centrals, respectively. The dashed lines indicate the median of each distribution.

sample in three populations: pre-processed central galaxies, pre-processed satellites, and in-situ processed satellites. Note that for the pre-processed central population, the mass of the host where the galaxies reside at the moment of processing is nearly the mass of the galaxy itself. From this panel we can clearly see that the three populations are well separated in the host mass distribution, regardless of the mass of the cluster. The median $\mathrm{M}_{\text {host }}$ of each population is indicated with dashed lines. As we can see, according to the criterion $\Gamma_{\mathrm{SFR}}^{\mathrm{SD}}$, central pre-processed galaxies tend to suffer their $\Gamma_{\mathrm{SFR}}^{\mathrm{SD}}$ in low-mass halos, preferentially in halos with total mass between $10^{10.5} \lesssim M_{\text {host }}\left[\mathrm{M}_{\odot}\right] \lesssim 10^{11.0}$. For galaxies pre-processed as satellites, $\Gamma_{\mathrm{SFR}}^{\mathrm{SD}}$ occurs in a large variety of halo masses, ranging between $10^{11} \lesssim \mathrm{M}_{\text {host }}\left[\mathrm{M}_{\odot}\right] \lesssim 10^{13.5}$, with a median near $10^{13.0} \mathrm{M}_{\text {host }}\left[\mathrm{M}_{\odot}\right]$ regardless of the mass of the cluster (the typical mass of galaxy groups). On the other hand, for the in-situ processed, it preferentially occurs in higher mass halos, with total masses larger than $10^{14.0} \mathrm{M}_{\text {host }}\left[\mathrm{M}_{\odot}\right]$.

To explore the relation between $\Gamma_{\mathrm{SFR}}^{\mathrm{SD}}$ and environment we compute, for the overall processed galaxy population, the distribution of total mass $\left(\mathrm{M}_{\text {galaxy }}\right)$ and the stellar mass fraction $\left(\mathrm{M}_{\star} / \mathrm{M}_{\text {galaxy }}\right)$ at the time they suffer their $\Gamma_{\mathrm{SFR}}^{\mathrm{SD}}$. These are shown on panels b) and c) of Figure 3, respectively. In general we find that in-situ processed galaxies tend to have a marginally larger $\mathrm{M}_{\text {galaxy }}$ than pre-processed galaxies. Interestingly, the difference in $\left(M_{\star} / M_{\text {galaxy }}\right)$ for these three populations is significantly more evident, with the central preprocessed galaxies showing the lowest stellar mass fractions. This is in agreement with the results shown in Figure 2, where we show that $\Gamma_{\text {SFR }}^{\text {SD }}$ for the in-situ population occurs at lower redshift, thus giving more time to these galaxies to grow in stellar mass. Note as well that there is a preference for pre-processing to occur in galaxies when they still remain as centrals, specially for the LMC bin, as shown by the green bars. We found that, for the pre-processed population, $54.07 \%$ in the HMC bin, $52.14 \%$ in the IMC, and $69.81 \%$ in the LMC were pre-processed as centrals.

As expected for central galaxies, the $\mathrm{M}_{\text {host }}$ and $\mathrm{M}_{\text {galaxy }}$ distributions are similar. In Figure 3, panel d), we show the distribution of stellar mass, $M_{\star}$, for all galaxies at the time of the first $R_{200}$ crossing. We can clearly see that the difference in $M_{\star}$ between in-situ and pre-processed galaxies is not only present at the time of $\Gamma_{\mathrm{SFR}}^{\mathrm{SD}}$, but pre-processed galaxies tend to arrive in the cluster with a significantly lower stellar mass. These results suggest that one of the strongest effects associated with this pre-processing is to limit the final stellar mass of satellites in galaxy clusters. As an example, in Figure 1 , Panel c, we show how the $\Gamma_{\mathrm{SFR}}^{\mathrm{SD}}$ significantly affects the 


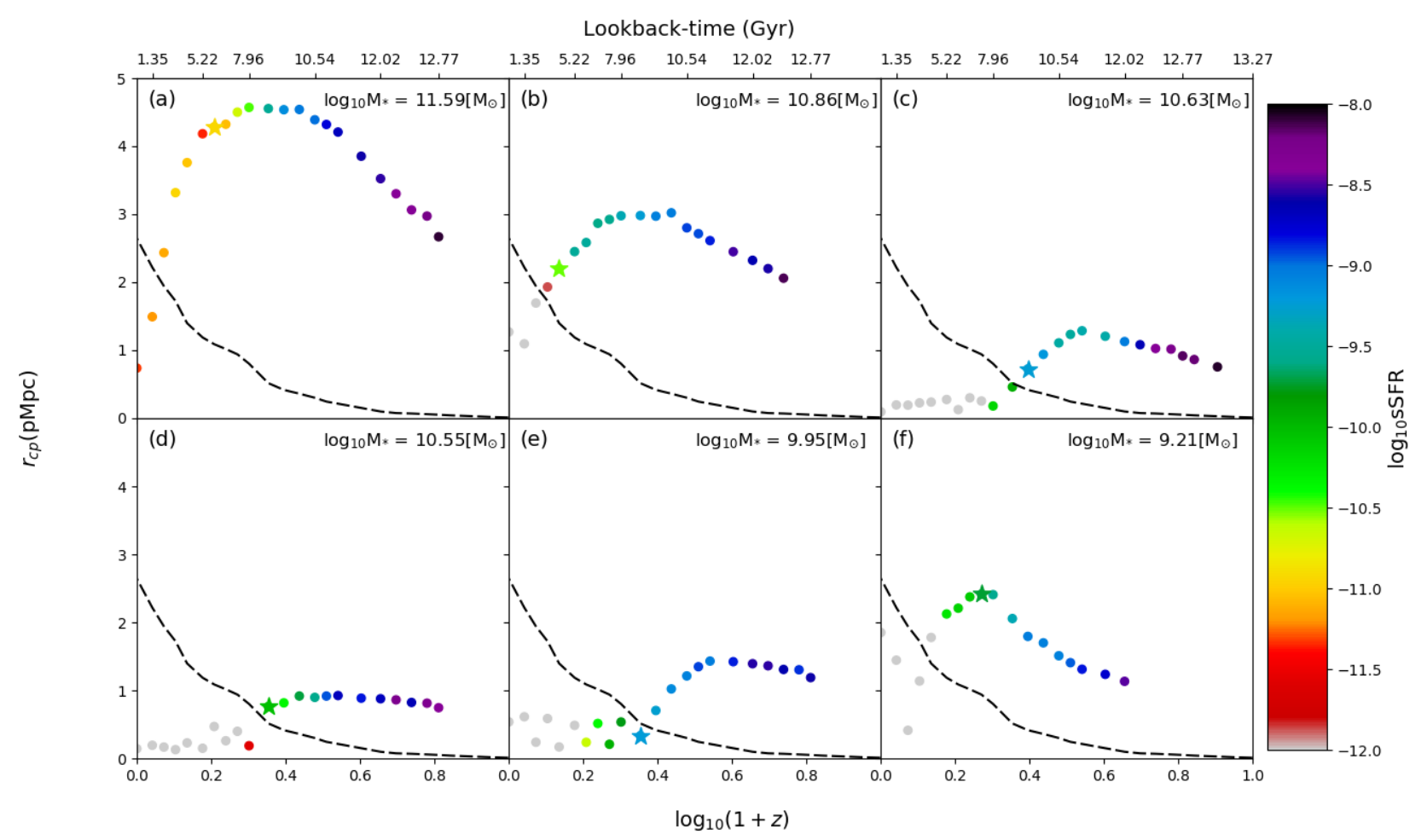

Figure 5. Time evolution of cluster-centric distance for a subset of four galaxies in our sample. In panel a) we show a galaxy pre-quenched as central, in panels b) and f) we have galaxies pre-quenched as satellite, and for panels c), d) and e), we have galaxies quenched in-situ. The color coding indicates the sSFR at each time. The dashed line shows the time evolution of the cluster $\mathrm{R}_{200}$ and the star shows the moment when the galaxies suffer their "processing" event. The label indicates the galaxy stellar mass at $z=0$.

subsequent growth of $\mathrm{M}_{\star}$ in a galaxy. For the pre-processed population, we have derived the time difference between the infall time, $t_{\text {inf }}$, and the pre-processing time, $t_{\text {proc }}$. In general, we find that $\left(t_{\text {proc }}-t_{\text {inf }}\right)$ is smaller for satellite galaxies than for centrals, and that this quantity grows with cluster mass. This result explains the difference in stellar mass ratio at the moment of the processing seen in Figure 3 for the pre-processed population. Central galaxies suffer their preprocessing earlier than the satellite sample and, despite the fact that both populations shows similar $\mathrm{M}_{\star}$ at the moment of the infall, those which had their strongest drop as centrals are more dark matter dominated.

It is clear from Figure 3 that centrals represent an important fraction of the pre-processed population, as they constitute $\gtrsim 50 \%$ of this population in any mass bin. In isolated and low-mass galaxies several mechanisms can significantly affect the star formation history and current star formation activity. Examples are photo-reionization, which limits their gas reservoir to form stars (Hopkins et al. 2014; Chan et al. 2018), or supernova feedback which, thanks to the injection of large amounts of kinetic energy into the intergalactic medium, can eject significant fractions of the available gas (Dekel \& Silk 1986; Davé et al. 2011; Biernacki \& Teyssier 2018). In addition, as shown by BenítezLlambay et al. (2013), ram-pressure stripping from the gas distribution within the cosmic web can efficiently remove the gas content of isolated low-mass galaxies. Pre-processing in galaxies that were not centrals at the time of $\Gamma_{\mathrm{SFR}}^{\mathrm{SD}}$ is gener- ally associated with ram-pressure stripping within the corresponding host. However, Figure 3 shows that the environment associated with a massive host galaxy plays a minor role in the pre-processing of low-mass galaxies.

As discussed before, the fraction of galaxies processed in-situ is rather low $(\lesssim 30 \%)$, and these galaxies tend to be more massive than the pre-processed population at the time of their corresponding $\Gamma_{\mathrm{SFR}}^{\mathrm{SD}}$. Their most significant drop in star formation activity took place within the $R_{200}$ of the main cluster. Thus, the main mechanisms acting are tidal and ram-pressure stripping within the cluster itself. This highlights the role played by the denser environment associated with galaxy clusters.

There is a small fraction $(<30 \%)$ of pre-processed galaxies for which $\Gamma_{\mathrm{SFR}}^{\mathrm{SD}}$ takes place in high-mass halos different from the main cluster. These halos correspond to objects that belong to massive galaxy-groups, in the mass range $10^{13.0} \lesssim \mathrm{M}_{\text {host }}\left[\mathrm{M}_{\odot}\right] \lesssim 10^{14.0}$, that are later accreted into the main cluster.

\subsection{Critical sSFR Selection Criterion}

In Section 4.1 we focused on the the properties of galaxies when they suffer their strongest drop in their star formation, $\Gamma_{\mathrm{SFR}}^{\mathrm{SD}}$. These drops do not necessarily result in the cessation of the star formation activity. Rather, as shown in Section 4.1, on average pre-processed galaxies arrive in the cluster with a significantly lower stellar mass than those 

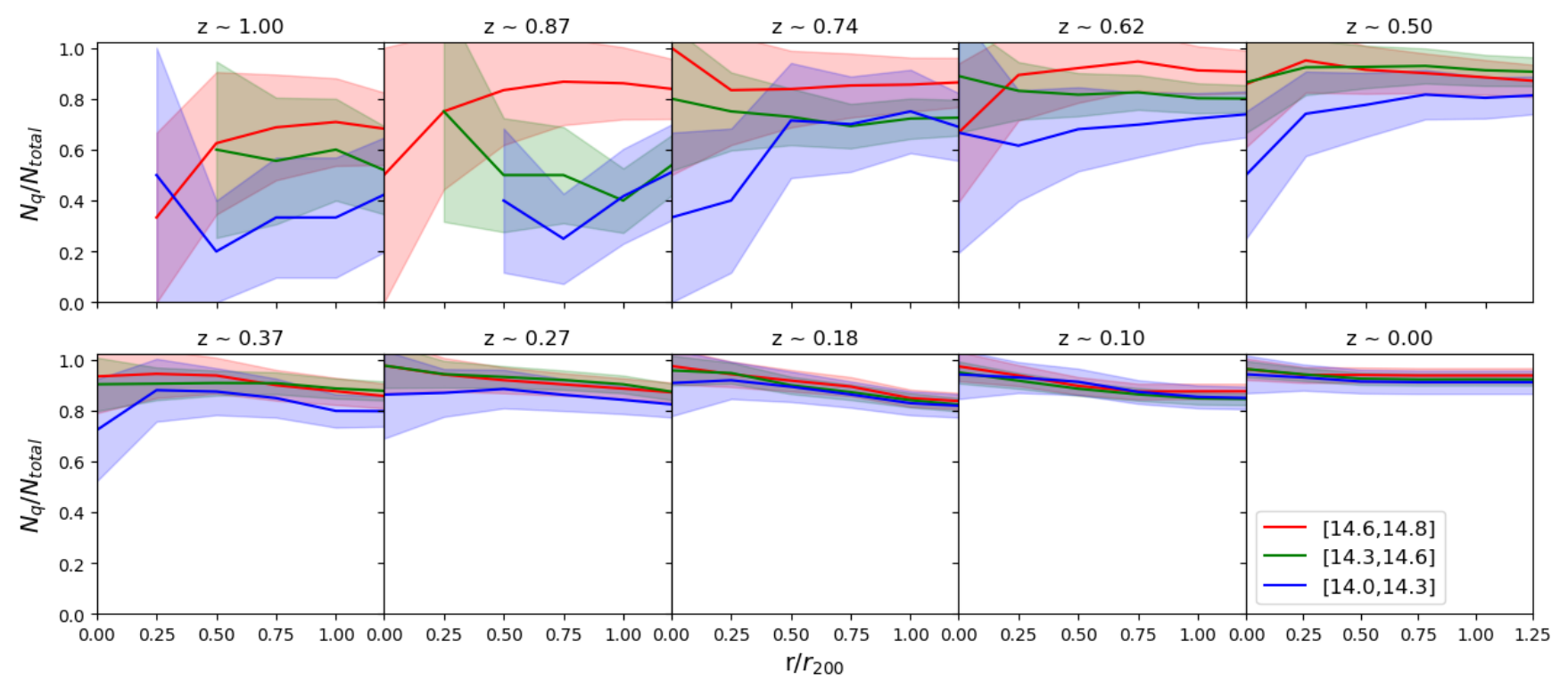

Figure 6. Time evolution of the cumulative fraction of quenched galaxies within the clusters' $R_{200}$. The red, green and blue lines show the results for the high, intermediate and low mass clusters, respectively. The shaded regions show Poissonian errors.

galaxies processed in-situ. Thus, instead of ceasing the star formation activity, an early $\Gamma_{\mathrm{SFR}}^{\mathrm{SD}}$ constrains the final galactic stellar mass.

In this Section we will focus on the moment when galaxies become effectively quenched. In $\mathrm{R}_{200}$ each cluster, we search for galaxies with sSFR values lower than $\mathrm{sSFR}^{\mathrm{Q}}$ defined in Section 3.2, and track their specific star formation history to identify the moment when this threshold is crossed. As before, we separate our galaxy sample in three bins according to cluster mass. The number of quenched galaxies in each bin is $\mathrm{N}_{\text {gal }}=780,1282$ and 374 for the HMC, IMC and LMC bins, respectively. Note that, in general, the number of quenched galaxies in each bin is $\lesssim 12 \%$ smaller than the number of galaxies that have suffered some type of processing.

In the left panels of Figure 4 we show the host mass distribution associated with each galaxy at the time in which they became quenched. As before, for galaxies that became quenched while being centrals (green bars), $\mathrm{M}_{\text {host }} \sim \mathrm{M}_{\text {galaxy }}$. Contrary to what is found with the $\Gamma_{\mathrm{SFR}}^{\mathrm{SD}}$ criterion, we find that, independently of the cluster mass bin, the vast majority of galaxies become quenched within massive hosts with $10^{13.5} \lesssim \mathrm{M}_{\text {host }}\left[\mathrm{M}_{\odot}\right] \lesssim 10^{14.5}$. This highlights the important role played by the denser environment of massive clusters on the overall quenching of their galaxy members. As an example we show, in Figure 5, the time evolution of the sSFR of six galaxies in our sample as they approach the central galaxy of one of our clusters. The dashed lines show the time evolution of the clusters $R_{200}$ and the color bar the sSFR of each galaxy. The star denotes the moment when the $\Gamma_{\mathrm{SFR}}^{\mathrm{SD}}$ takes place. Note that galaxies in panel a) reach their quenching state as centrals. Also, it is interesting to note that the quenching state is reached as a consequence of their $\Gamma_{\mathrm{SFR}}^{\mathrm{SD}}$. For galaxies in panels b) and f), they reach their quenching state as satellites before they were accreted by the cluster, and galaxies in panels c) d) and e) are quenched inside the cluster $\mathrm{R}_{200}$. Also, in any case, as galaxies approach the cluster center, their sSFR slowly decreases. However, the change in sSFR just after the first $R_{200}$ crossing is significantly more abrupt, in some cases rapidly resulting in quenching. On the other hand, galaxies that quenched in low-mass halos, i.e. $10^{10.0} \lesssim \mathrm{M}_{\text {host }}\left[\mathrm{M}_{\odot}\right] \lesssim 10^{11.0}$, did it as centrals, highlighting the regime where internal quenching processes are most relevant.

The red bars on Figure 4 indicate the distributions of the in-situ quenched galaxies population. Interestingly, we find that the fraction of galaxies that arrived in the cluster already quenched (i.e., pre-quenched population) increases with cluster mass. For comparison we find $73 \%$ of the galaxies were quenched in-situ in the LMC bin, but only $45 \%$ in the HMC bin. This apparent relation between the fraction of pre-quenched galaxies with cluster mass is further explored below. As in the case of the $\Gamma_{\mathrm{SFR}}^{\mathrm{SD}}$ criterion, we find the total mass distribution of pre- and in-situ quenched galaxies to be very similar (medium-left panels), but they show a significant offset on their stellar masses at the moment of quenching (medium-right panels). As expected, we find that most pre-quenched galaxies $(\sim 95 \%)$ have also been preprocessed, indicating the important role played by the preprocessing in the quenching of low-mass objects. Panel d) of Figure 4 shows the distribution of $\left(t_{\mathrm{q}}-t_{\text {inf }}\right)$, where $t_{\mathrm{q}}$ represents the galaxy quenching time. We find a relation between $t_{\mathrm{q}}$ and cluster mass for the pre-quenched population, where the high mass bin presents bigger differences between both times. This is a result of the hierarchical scenario; i.e., bigger clusters accrete bigger structures and, thus, environmental effects are more significant since earlier epochs. In general for the in-situ quenched population, we find no difference in $\left(t_{\mathrm{q}}-t_{\text {inf }}\right)$, between the different mass bins, highlighting the role of the virial-radius crossing in the star formation quenching of galaxies.

In figure 6 we show the time evolution of the cumula- 
tive fraction of quenched galaxies, $N_{\mathrm{q}} / N_{\text {total }}$, as a function of cluster-centric distance. Here, $N_{\mathrm{q}}$ represents the number of quenched galaxies within a given radius, $R$, and $N_{\text {total }}$ the total number of galaxies within the same distance. The different lines correspond to the different cluster mass bins. Interestingly, we see that at early times, between $z \sim 1$ and $z \sim 0.5$, the fraction of quenched galaxies grows towards the cluster outskirts. However, at later times this trend reverses, showing a decreasing fraction of quenched galaxies with distance. During the last decade, surveys such as WINGS (Cava et al. 2017) and SAMI (Brough et al. 2017) have shown that:

(i) the fraction of quenched galaxies grows towards $z=0$. This is attributed to the environment having more time to act on cluster galaxies;

(ii) the fraction of quenched galaxies decreases with cluster-centric distance. Thanks to the denser environments that can be found in the inner cluster region, galaxies, especially those with lower masses, can be more efficiently depleted of their gas reservoir.

Our results are in good agreement with these observations. We have previously highlighted a correlation between the fraction of pre-quenched galaxies and cluster mass. We further explore this correlation in Figure 7. Here we show how the cumulative fraction of quenched galaxies, with respect to the total number of all galaxies that can be found within $\mathrm{R}_{200}$ at $z=0$, grows as a function of the normalized time, $t-t_{\text {infall }}$. To obtain this plot, we first compute for each galaxy within $R_{200}$ at $z=0$ the time when it crossed $R_{200}$ for the first time. Second, for each galaxy we define the variable $t-t_{\text {infall }}$ and identify the moment when it became quenched on this new time scale. Finally, we compute the cumulative quenched galaxy fraction as a function of $t-t_{\text {infall }}$. This figure allows us to study how the fraction of quenched galaxies changes as a function of the time they remain either outside (negative $t-t_{\text {infall }}$ ) or inside (positive $\left.t-t_{\text {infall }}\right)$ the cluster's $\mathrm{R}_{200}$. The different lines are associated with the galaxy populations of different clusters. The colors indicate the mass of each cluster at $z=0$. Note that, in all clusters, the fraction of quenched galaxies slowly grows as galaxies approach the cluster's $\mathrm{R}_{200}$, again highlighting the role of pre-processing. Interestingly, there is a change in the slope of this cumulative function around the time of the first $R_{200}$ crossing, i.e. $-1 \mathrm{Gyr} \lesssim t-t_{\text {infall }} \lesssim 1 \mathrm{Gyr}$. During this period, the fraction of quenched galaxies raises more rapidly than during any other epoch. This is in agreement with the behaviour of the sSFR observed in Figure 5, and clearly displays the role played by the cluster's environment. We can also observe a large dispersion in the fraction of galaxies that arrive quenched at the cluster's $\mathrm{R}_{200}$, with values that go from 20 to $60 \%$. More importantly, this fraction shows a dependency with final cluster mass, with larger values for more massive clusters.

To study the origin of this trend we compute the mass distribution of the structures, $\mathrm{M}_{\text {host }}$, where the quenched galaxy population at $z=0$ were located at the snapshot before their first $\mathrm{R}_{200}$ crossing. This is shown in Figure 8, panels a). As before, each row corresponds to the results obtained from a different cluster mass bin. The blue bars indicate the fraction of pre-quenched galaxies, while the white bars show all the quenched galaxies found within the cluster at $z=0$. The dashed lines indicate the median for the

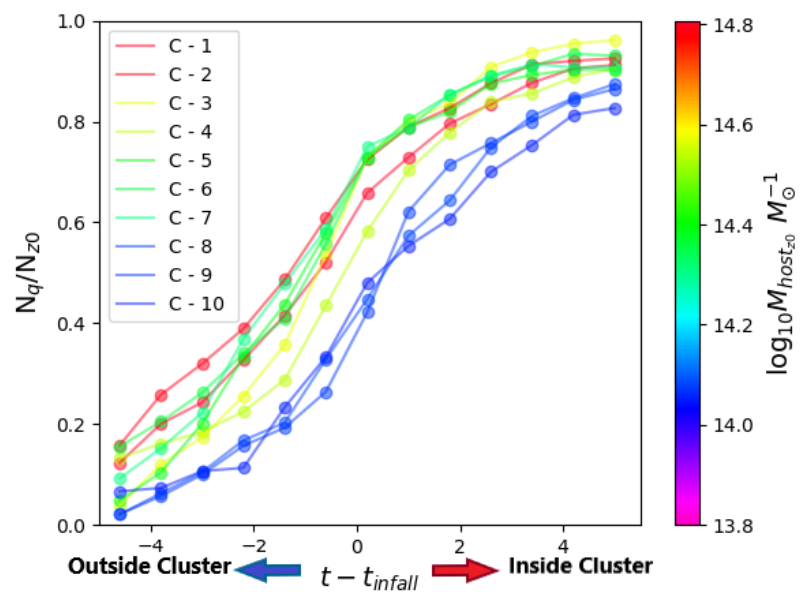

Figure 7. Cumulative fraction of quenched galaxies as a function of the normalized time scale, $t-t_{\text {infall }}$. The infall time $t_{\text {infall }}$ is computed for each individual galaxy. The color coding indicates the total mass of each cluster at $z=0$. Negative (positive) $t-t_{\text {infall }}$ corresponds to periods of time when galaxies are located outside (inside) the cluster's $R_{200}$.

pre-quenched population. Interestingly, pre-quenched galaxies on the LMC bin tend to arrive in lower mass structures than in the rest of the cluster mass bins. However, no significant difference is observed in both the distribution of total $\left(\mathrm{M}_{\text {galaxy }}\right)$ and stellar masses $\left(\mathrm{M}_{\star}\right)$ of the pre-quenched galaxy populations at infall, shown in panels b) and c), respectively.

Our results indicate that the larger fraction of prequenched galaxies in larger mass clusters is the result of the hierarchical nature of the $\Lambda \mathrm{CDM}$ cosmological model used in this work, in which larger mass object can accrete more massive substructures. These more massive substructures are naturally more efficient in quenching their own galaxy satellite population, thus resulting in a larger fraction of pre-quenched galaxies at $z=0$.

\section{DISCUSSION AND CONCLUSIONS}

In this paper we have presented a study of the different environmental-quenching and processing scenarios undergone by the satellite galaxies of the ten most massive clusters in the state-of-the-art EAGLE hydrodynamical simulation. Two different criteria were defined to explore the different processes that significantly affect the SFR of these galaxies along their history. Our goal is to quantify and characterize the role played by the environment in these processes.

For the instantaneous strongest drop in SFR, we find that the majority of galaxies suffer their $\Gamma_{\mathrm{SFR}}^{\mathrm{SD}}$ outside the cluster's $R_{200}$ (pre-processed fraction $\gtrsim 60 \%$ ). This fraction grows with cluster mass. We find that there is no correlation between the strength of the $\Gamma_{\mathrm{SFR}}^{\mathrm{SD}}$ and the time at which it occurs, nor a preferential redshift for it to happen. Nonetheless, for galaxies processed in-situ, $\Gamma_{\mathrm{SFR}}^{\mathrm{SD}}$ tends to happen at lower redshift than for the pre-processed population. In terms of the environment, while in-situ processing mainly occurs in massive hosts, pre-processing shows a strong pref- 
(a)

(b)

(c)

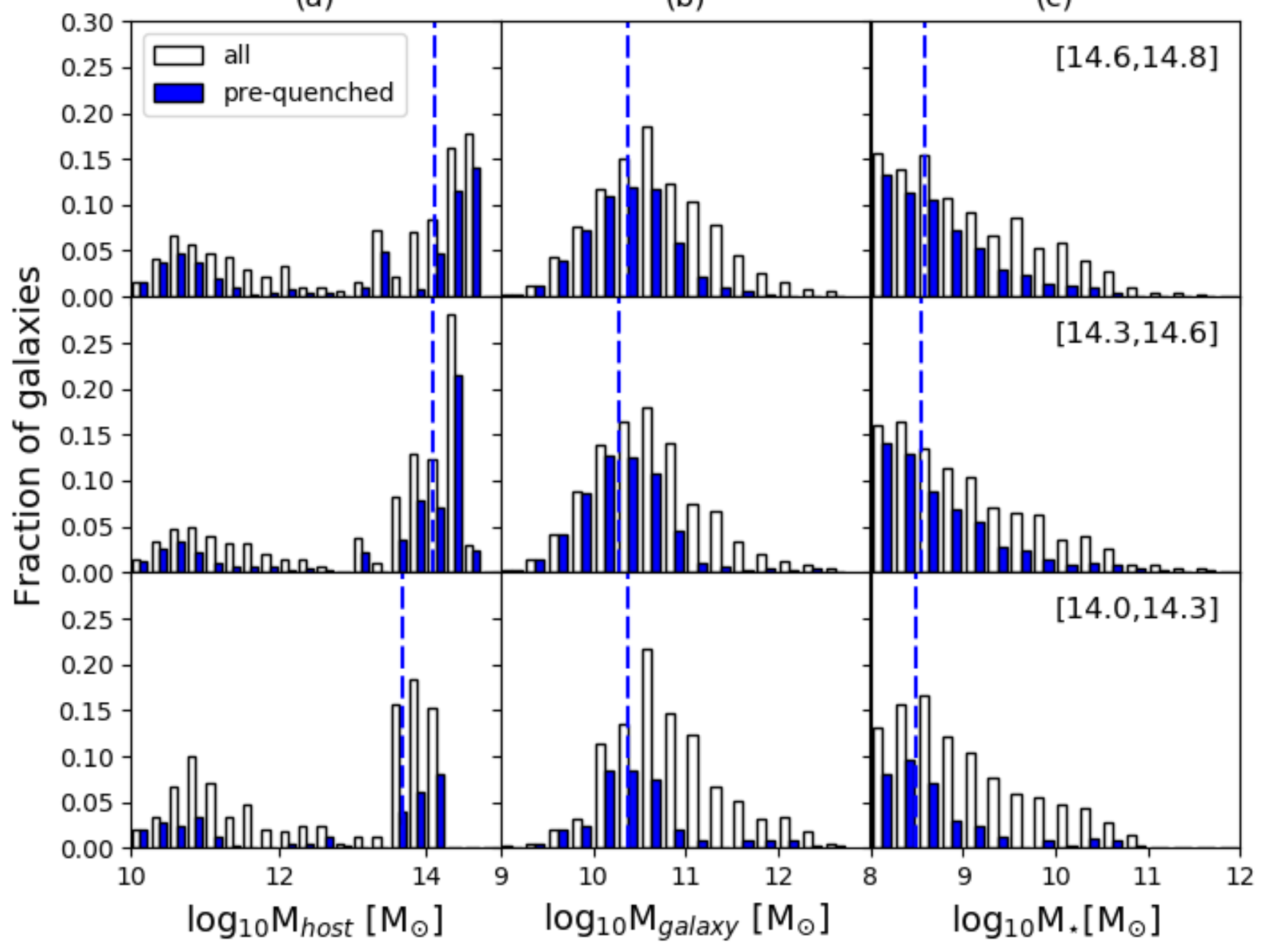

Figure 8. Mass distribution of galaxies and their hosts at the moment before crossing the cluster's $\mathrm{R}_{200}$. Each row shows the results obtained after stacking the distribution of galaxies associated with clusters within different mass ranges. Column (a) shows the mass distribution of the host of each galaxy. Column (b) shows the distribution of galaxy total mass. Column (c) shows the distributions of stellar mass. The blue bars correspond to the galaxies quenched before the first infall and the white bars correspond to all galaxies in our sample. Dashed lines correspond to the median of the pre-quenched population.

erence to take place in galaxies that are either low mass and central $\left(10^{10.5} \lesssim \mathrm{M}_{\text {host }}\left[\mathrm{M}_{\odot}\right] \lesssim 10^{11.0}\right)$ or that belong to low-to-middle mass hosts $\left(10^{11} \lesssim \mathrm{M}_{\text {host }}\left[\mathrm{M}_{\odot}\right] \lesssim 10^{13.5}\right)$. Our results are in good agreement with those published by Bianconi et al. (2018), who observationally studied a sample of 23 massive clusters $\left(\mathrm{M}_{200}=10^{15.0}\left[\mathrm{M}_{\odot}\right]\right)$ with 34 infalling groups $\left(\log _{10} \mathrm{M}_{\star}\left[\mathrm{M}_{\odot}\right]=10.75\right)$, located in outer cluster regions. They found that at cluster-centric distances $\mathrm{R} \sim 1.3 \mathrm{R}_{200}$ the fraction of star-forming galaxies in infalling groups is half of that in the clusters. According to this, Bianconi et al. (2018) suggest that the pre-processing in groups is the responsible for these results.

Interestingly, for galaxies with similar total mass, at the time of arrival in the main cluster, the in-situ processed population shows in general a larger stellar mass than those pre-processed. This highlights the important role of preprocessing in limiting the star formation activity of low-mass galaxies. The origin of this pre-processing event can be ex- plained by a variety of different internal mechanisms such as supernova feedback, photo-reionization, interactions and starburst phases. Unfortunately, due to the poor time and spatial resolution available with this simulation, it is too hard to identify what the main mechanism acting on each galaxy is. In addition, ram-pressure from the cosmic web can also cause an accelerated depletion of the gas reservoirs in low mass galaxies, producing abrupt changes in their star formation (Benítez-Llambay et al. 2013).

In the case of the Critical sSFR criterion, contrary to our results for $\Gamma_{\mathrm{SFR}}^{\mathrm{SD}}$, we find that quenching presents a strong preference for high-mass halos to take place. This is a strong indicator that dense environments promote the definitive cessation of the star formation.

Our results are in agreement with the observations presented by Olave-Rojas et al. (2018), who find that the fraction of high-mass $\left(\mathrm{M}_{\star} \geq 10^{10.5}\left[\mathrm{M}_{\odot}\right]\right)$ red (i.e. passive) galaxies in clusters (i.e quenched in-situ) is higher than the frac- 
tion of high-mass red galaxies in accreted groups (i.e. prequenched). We find that most of the pre-quenched galaxies ( $\gtrsim 95 \%$ ) have also been pre-processed, evidencing the importance of pre-processing in the quenching of low-mass galaxies. In general we find a slight preference for pre-quenching to take place at earlier times compared with quenching insitu. The difference in the median of the quenching time distribution is only of the order of 1 to 2 Gyr. As a function of cluster-centric distance, close to $z=0$ the fraction of quenched galaxies grows toward the cluster center. This is in good agreement with the results obtained from observational studies based on different surveys such as WINGS (Cava et al. 2017) and SAMI (Brough et al. 2017). However, at earlier times, between $z \sim 1$ and $z \sim 0.5$, this trend reverts, showing a fraction of quenched galaxies that grows towards the cluster outskirts.

In general, we find that in comparison to the in-situ quenched population, on average pre-quenched galaxies have lower stellar-masses. This result appears to be in disagreement with those presented by Hou et al. (2014) who found that, independent of galaxy mass, the fraction of quiescent galaxies is higher in groups than in the clusters and field. However, we can reconcile our findings with those of Hou et al. (2014) by noting that those authors only studied galaxies with stellar masses in the range $9.5<\log _{10} \mathrm{M}_{\star}\left[\mathrm{M}_{\odot}\right]<10.5$ and with $10^{12.0} \leq \mathrm{M}_{\text {halo }}\left[\mathrm{M}_{\odot}\right] \leq 10^{14.0}$. In these massive and dense substructures the environmental quenching effects are stronger.

We find a sharp rise in the fraction quenched satellites at the time of the first infall, highlighting the role played by the dense cluster environment. It is interesting to note that, although galaxies prefer denser environments to reach their quenching state, the fraction of pre-quenched galaxies in our sample grows with the total mass of the cluster at $z=0$. We find that $73 \%$ of galaxies were quenched in-situ in the low-mass clusters, but only $45 \%$ were quenched in-situ for the high-mass clusters. To explain why high-mass clusters show higher fractions of pre-quenched galaxies, we explore the mass distribution of the structures where the cluster satellite galaxies reside at the moment of accretion. We find that high-mass clusters preferentially accrete their satellites through structures and groups that are significantly more massive than those accreted by low-mass clusters. This is a direct consequence of the hierarchical cosmological model used in these simulations. More massive clusters tend to accrete more massive substructures. Due to their own intracluster dense environments, these massive substructures arrive in the clusters with their satellite population already quenched.

Cora et al. (2018a) explored the quenching time of galaxies, and the relevance of the environment on this process, using the semi-analytic model SAG (Cora et al. 2018b). A criterion similar to our sSFR threshold was imposed. According to their results, environmental effects dominate the star formation quenching of low-mass satellite galaxies $\left(M_{\star}\right.$ $<10^{10.1}\left[\mathrm{M}_{\odot}\right]$. These results are in good agreement with our results. Panels a) and c) of Figure 4 show that we also find an important fraction of low-stellar mass galaxies that are quenched within the cluster's $\mathrm{R}_{200}$. Note that a significant fraction of the low-stellar mass galaxies that arrive in the cluster as quenched galaxies were actually quenched in the dense environments of massive groups. This exemplifies the relevance of the environment in the quenching of the cluster satellite population.

We also find that there is a fraction of low-stellar mass galaxies that are quenched as centrals. According to BenítezLlambay et al. (2013), this can be explained through a combination of different mechanisms that are acting simultaneously on dwarf galaxies. Processes such as supernova feedback and photo-reionization can reheat the cool gas of these galaxies inducing the quenching of their star formation activity, a scenario commonly referred to as mass quenching. In addition, as previously discussed, ram-pressure stripping taking plance within the cosmic web filaments can also deplete the gas reservoir of dwarf galaxies, producing a quenching state due to the environment.

As we mentioned before, due to the limtied number of snapshots available in the simulation, we do not have the capabilities to separate and distinguish the different overlaping processes that are influencing the star formation history of the galaxies. In a follow-up project we plan to explore these different mechanisms using more detailed hydrodynamical simulations from the C-EAGLE project. These simulations provide us with a great improvement in temporal resolution, with a temporal resolution for particles of $\tilde{1} 25 \mathrm{Myr}$ and $\tilde{2} 5$ Myr for three particular intervals of redshift ([0-1],[4-5],[7$8]$ ), and 500 Myr for group catalogues (Barnes et al. 2017; Bahé et al. 2017). Since this simulation suite also counts with a sample of 30 clusters with a $\mathrm{M}_{200}$ in the range between of $10^{14.0}<\mathrm{M}_{200}\left[\mathrm{M}_{\odot}\right]<10^{15.4}$, this study will also allow us to explore in more detail the dependency between cluster mass and fraction of pre-quenched galaxies.

\section{ACKNOWLEDGEMENTS}

We thank Daniel Hernandez, Catalina Labayru, Ciria Lima, Antonella Monachesi and Catalina Mora for useful discussions and comments. We also want to thank the anonymous referee for his/her many insightful comments, that greatly improved the quality of the manuscript. We acknowledge the Virgo Consortium for making their simulation data available. The EAGLE simulations were performed using the DiRAC-2 facility at Durham, managed by the ICC, and the PRACE facility Curie based in France at TGCC, CEA, Bruyeres-le-Chatel. We also thank the support given by the "Vicerrectoría de Investigación de la Univesidad de La Serena" for the support given by the program "Apoyo al fortalecimiento de grupos de investigación". DP also acknowledges financial support through the fellowship "Becas Doctorales Institucionales ULS", granted by the "Vicerrectoría de Investigación y Postgrado de la Universidad de La Serena. DP also thanks the hospitality of PUC during the stay at the university. DP and FAG acknowledge financial support from the Max Planck Society through a Partner Group grant. FAG acknowledges financial support from CONICYT through the project FONDECYT Regular Nr. 1181264. D.O-R acknowledges the financial support provided by CONICYT-PCHA through a PhD Scholarship, "Beca Doctorado Nacional Año 2015", under contract 2015-21150415. P.C. acknowledges the support provided by FONDECYT postdoctoral research grant no 3160375. NP acknowlodges the support from BASAL AFB-170002 CATA, CONICYT Anillo-1477 and Fondecyt Regular 1150300. R.D. 
gratefully acknowledges support from the Chilean Centro de Excelencia en Astrofísica y Tecnologías Afines (CATA) BASAL grant AFB-170002.

\section{REFERENCES}

Abadi M. G., Moore B., Bower R. G., 1999, MNRAS, 308, 947 Bahé Y. M., et al., 2017, MNRAS, 470, 4186

Baldry I. K., Balogh M. L., Bower R. G., Glazebrook K., Nichol R. C., Bamford S. P., Budavari T., 2006, MNRAS, 373, 469

Balogh M. L., et al., 2007, MNRAS, 374, 1169

Balogh M. L., et al., 2016, MNRAS, 456, 4364

Barnes J. E., Hernquist L., 1996, ApJ, 471, 115

Barnes D. J., et al., 2017, MNRAS, 471, 1088

Benítez-Llambay A., Navarro J. F., Abadi M. G., Gottlöber S., Yepes G., Hoffman Y., Steinmetz M., 2013, ApJ, 763, L41

Bianconi M., Smith G. P., Haines C. P., McGee S. L., Finoguenov A., Egami E., 2018, MNRAS, 473, L79

Biernacki P., Teyssier R., 2018, MNRAS, 475, 5688

Boselli A., Gavazzi G., 2006, PASP, 118, 517

Boselli A., et al., 2005, ApJ, 629, L29

Bravo-Alfaro H., Cayatte V., van Gorkom J. H., Balkowski C., 2000, AJ, 119, 580

Brough S., et al., 2017, ApJ, 844, 59

Butcher H., Oemler Jr. A., 1984, ApJ, 285, 426

Byrd G., Valtonen M., 1990, ApJ, 350, 89

Cantalupo S., 2010, MNRAS, 403, L16

Cassata P., et al., 2008, A\&A, 483, L39

Cava A., et al., 2017, A\&A, 606, A108

Cayatte V., van Gorkom J. H., Balkowski C., Kotanyi C., 1990, AJ, 100, 604

Chabrier G., 2003, PASP, 115, 763

Chan T. K., Kereš D., Wetzel A., Hopkins P. F., Faucher-Giguère C.-A., El-Badry K., Garrison-Kimmel S., Boylan-Kolchin M., 2018, MNRAS, 478, 906

Chester C., Roberts M. S., 1964, AJ, 69, 635

Cora S. A., Hough T., Vega-Martínez C. A., Orsi Á., 2018a, preprint, (arXiv:1801.03884)

Cora S. A., et al., 2018b, MNRAS, 479, 2

Crain R. A., et al., 2015, MNRAS, 450, 1937

Croton D. J., et al., 2006, MNRAS, 365, 11

Cybulski R., Yun M. S., Fazio G. G., Gutermuth R. A., 2014, MNRAS, 439, 3564

Dalla Vecchia C., Schaye J., 2012, MNRAS, 426, 140

Davé R., Oppenheimer B. D., Finlator K., 2011, MNRAS, 415, 11

Davis M., Efstathiou G., Frenk C. S., White S. D. M., 1985, ApJ, 292,371

De Lucia G., Weinmann S., Poggianti B. M., Aragón-Salamanca A., Zaritsky D., 2012, MNRAS, 423, 1277

Dekel A., Birnboim Y., 2008, MNRAS, 383, 119

Dekel A., Silk J., 1986, ApJ, 303, 39

Desai V., et al., 2007, ApJ, 660, 1151

Dolag K., Borgani S., Murante G., Springel V., 2009, MNRAS, 399, 497

Dressler A., 1980, ApJ, 236, 351

Dressler A., 1984, ARA\&A, 22, 185

Dressler A., et al., 1997, ApJ, 490, 577

Dressler A., Oemler Jr. A., Poggianti B. M., Gladders M. D., Abramson L., Vulcani B., 2013, ApJ, 770, 62

Efstathiou G., 2000, MNRAS, 317, 697

Faber S. M., 1973, ApJ, 179, 731

Fasano G., Poggianti B. M., Couch W. J., Bettoni D., Kjærgaard P., Moles M., 2000, ApJ, 542, 673

Fujita Y., 2004, PASJ, 56, 29

Furlong M., et al., 2015, MNRAS, 450, 4486

Furlong M., et al., 2017, MNRAS, 465, 722
Gavazzi G., Fumagalli M., Cucciati O., Boselli A., 2010, A\&A, 517, A73

Gray M. E., Wolf C., Meisenheimer K., Taylor A., Dye S., Borch A., Kleinheinrich M., 2004, MNRAS, 347, L73

Gunn J. E., Gott III J. R., 1972, ApJ, 176, 1

Haardt F., Madau P., 2001, in Neumann D. M., Tran J. T. V., eds, Clusters of Galaxies and the High Redshift Universe Observed in X-rays. p. 64 (arXiv:astro-ph/0106018)

Haines C. P., et al., 2013, ApJ, 775, 126

Haines C. P., et al., 2015, ApJ, 806, 101

Hashimoto Y., Oemler Jr. A., Lin H., Tucker D. L., 1998, ApJ, 499,589

Hogg D. W., et al., 2004, ApJ, 601, L29

Hopkins P. F., Kereš D., Oñorbe J., Faucher-Giguère C.-A., Quataert E., Murray N., Bullock J. S., 2014, MNRAS, 445, 581

Hou A., Parker L. C., Harris W. E., 2014, MNRAS, 442, 406

Jaffé Y. L., Smith R., Candlish G. N., Poggianti B. M., Sheen Y.-K., Verheijen M. A. W., 2015, MNRAS, 448, 1715

Jaffé Y. L., et al., 2016, MNRAS, 461, 1202

Just D. W., Zaritsky D., Sand D. J., Desai V., Rudnick G., 2010, ApJ, 711, 192

Katsianis A., et al., 2017, MNRAS, 472, 919

Kauffmann G., White S. D. M., Heckman T. M., Ménard B., Brinchmann J., Charlot S., Tremonti C., Brinkmann J., 2004, MNRAS, 353, 713

Kennicutt Jr. R. C., 1998, ApJ, 498, 541

Kodama T., Smail I., Nakata F., Okamura S., Bower R. G., 2001, ApJ, 562, L9

Lewis I., et al., 2002, MNRAS, 334, 673

McAlpine S., et al., 2016, Astronomy and Computing, 15, 72

McGee S. L., Balogh M. L., Bower R. G., Font A. S., McCarthy I. G., 2009, MNRAS, 400, 937

Miller R. H., 1986, A\&A, 167, 41

Moore B., Katz N., Lake G., Dressler A., Oemler A., 1996, Nature, 379,613

Moore B., Lake G., Quinn T., Stadel J., 1999, MNRAS, 304, 465

Moran S. M., Ellis R. S., Treu T., Smith G. P., Rich R. M., Smail I., 2007, ApJ, 671, 1503

Morgan W. W., Mayall N. U., 1957, PASP, 69, 291

Muzzin A., et al., 2012, ApJ, 746, 188

Nantais J. B., et al., 2016, A\&A, 592, A161

Nelson D., et al., 2018, MNRAS, 475, 624

Oesch P. A., et al., 2016, ApJ, 819, 129

Olave-Rojas D., Cerulo P., Demarco R., Jaffé Y. L., Mercurio A., Rosati P., Balestra I., Nonino M., 2018, MNRAS, 479, 2328

Overzier R. A., 2016, A\&ARv, 24, 14

Peng Y.-j., et al., 2010, ApJ, 721, 193

Peng Y.-j., Lilly S. J., Renzini A., Carollo M., 2012, ApJ, 757, 4

Planck Collaboration et al., 2014, A\&A, 566, A54

Poggianti B. M., Smail I., Dressler A., Couch W. J., Barger A. J., Butcher H., Ellis R. S., Oemler Jr. A., 1999, ApJ, 518, 576

Poggianti B. M., et al., 2001, ApJ, 562, 689

Poggianti B. M., et al., 2006, ApJ, 642, 188

Quilis V., Moore B., Bower R., 2000, Science, 288, 1617

Roberts M. S., Haynes M., 1994, in Meylan G., Prugniel P., eds, European Southern Observatory Conference and Workshop Proceedings Vol. 49, European Southern Observatory Conference and Workshop Proceedings. p. 197

Schaye J., 2004, ApJ, 609, 667

Schaye J., Dalla Vecchia C., 2008, MNRAS, 383, 1210

Schaye J., et al., 2015, MNRAS, 446, 521

Smail I., Ellis R. S., Dressler A., Couch W. J., Oemler A., Sharples R. M., Butcher H., 1997, ApJ, 479, 70

Springel V., 2005, MNRAS, 364, 1105

Springel V., White S. D. M., Tormen G., Kauffmann G., 2001, MNRAS, 328, 726

Strateva I., et al., 2001, AJ, 122, 1861 
Tescari E., et al., 2018, MNRAS, 473, 380

Toomre A., Toomre J., 1972, in Bulletin of the American Astronomical Society. p. 214

Trayford J. W., et al., 2015, MNRAS, 452, 2879

Trayford J. W., Theuns T., Bower R. G., Crain R. A., Lagos C. d. P., Schaller M., Schaye J., 2016, MNRAS, 460, 3925

Treu T., Ellis R. S., Kneib J.-P., Dressler A., Smail I., Czoske O., Oemler A., Natarajan P., 2003, ApJ, 591, 53

Vijayaraghavan R., Ricker P. M., 2013, MNRAS, 435, 2713

Vollmer B., Cayatte V., Balkowski C., Duschl W. J., 2001, ApJ, 561,708

Walker I. R., Mihos J. C., Hernquist L., 1996, ApJ, 460, 121

Weinmann S. M., Kauffmann G., von der Linden A., De Lucia G., 2010, MNRAS, 406, 2249

Wetzel A. R., Tinker J. L., Conroy C., 2012, MNRAS, 424, 232

Wetzel A. R., Tinker J. L., Conroy C., van den Bosch F. C., 2013, MNRAS, 432, 336

Wiersma R. P. C., Schaye J., Smith B. D., 2009a, MNRAS, 393, 99

Wiersma R. P. C., Schaye J., Theuns T., Dalla Vecchia C., Tornatore L., 2009b, MNRAS, 399, 574

Zabludoff A. I., Mulchaey J. S., 1998, ApJ, 498, L5

van den Bosch F. C., Aquino D., Yang X., Mo H. J., Pasquali A., McIntosh D. H., Weinmann S. M., Kang X., 2008, MNRAS, 387,79

This paper has been typeset from a $\mathrm{T}_{\mathrm{E}} \mathrm{X} / \mathrm{LAT}_{\mathrm{E}} \mathrm{X}$ file prepared by the author. 